\title{
Ancora a proposito di badante
}

\author{
Massimo Fanfani
}

PUBBLICATO: 31 MARZO 2019

Gettando un "Primo sguardo sul secondo millennio" della sempre risorgente questione della lingua, Claudio Marazzini ha ragione da vendere nel dedicare un intero capitolo al "Politicamente (s)corretto", il subdolo purismo ideologico della postmodernità, che corrode dall'interno il sistema semantico-lessicale (e perfino morfologico) delle lingue, asfaltandone storie, caratteri, particolarità (Claudio Marazzini, Da Dante alle lingue del Web. Otto secoli di dibattiti sullitaliano, Roma, Carocci, 2012, pp. 251-258). Uno dei settori in cui il cosiddetto "politicamente corretto" cerca di raddrizzare le presunte storture del linguaggio contemporaneo è quello delle parole che paiono o sono andate incontro a scadimento semantico: parole da sostituire senza eccezione con termini neutri o positivi, spesso nient'altro che eufemistiche foglie di fico, specie quando la sostanza non cambia. E così, per fare un noto esempio, invece di onorare il lavoro di chi già prima dell'alba simpegna a tener pulite le strade su cui cammineremo (un lavoro che ha la stessa dignità di quello del chirurgo o dell'ingegnere), si continua a riservargli scarsa considerazione, mentre lo si riveste di casacche sempre più sgargianti e lo si ribattezza sempre daccapo: scopino, spazzino, netturbino, operatore ecologico (raccoglitore porta a porta, separatore (di rifiuti), addetto alla spazzatrice, ecc.).

Fra le parole sulla graticola del postpurismo ideologico Marazzini si sofferma anche subadante, ritenuta offensiva dalle associazioni professionali, che vorrebbero sostituirle il più banale sintagma assistente familiare, "invocando la classificazione burocratica ISTAT delle professioni, illudendosi che l'ISTAT possa dettare le regole della lingua comune" (p. 255). Marazzini riporta anche un'interessante lettera di uno studente di un corso per operatori socio-sanitari, corso in cui è stato raccomandato di usare assistente familiare evitando la parola badante, propria un tempo di chi badava il bestiame, e di non pronunziarla mai agli esami, pena la bocciatura.

Davanti alla testimonianza di una tale censura si può ben comprendere la reazione di Marazzini: «È arbitrario riportare l'origine di "badante" al contesto della custodia di animali, quando l'origine è probabilmente una forma dialettale legata a usi familiari (cosi il romagnolo abadanta, badanta; il lessico romagnolo registra fin dall'Ottocento abadé nel senso di "aver cura di uno", per esempio badare a padri e fratelli). Del resto "badare" per "aver cura" esiste anche in toscano e in italiano letterario: si può "badare alle pecore", ma anche "badare ai fatti propri" e "badare alle parole che si dicono". Anche in questo caso, fantasie ed elucubrazioni arbitrarie scaricano sulle parole responsabilità che non esistono; tuttavia l'azione repressiva dei censori e inquisitori "politicamente corretti", come si evince dalla lettera, si esercita in forma molto minacciosa ed efficace. Si tratta di vedere se il burocratismo "assistente famigliare", asettico e poco trasparente, che contende la propria area semantica ad altri analoghi burocratismi indistinguibili per il parlante comune, ma tra loro assolutamente diversi in base a una logica sindacale e amministrativa (ausiliario socio-assistenziale, ausiliario di assistenza per anziani, operatore addetto all'assistenza, ecc.), potrà avere una ricaduta sull'uso linguistico dell'intera collettività» (pp. 257-258).

Nell'indicare l'origine romagnola di badante si coglie effettivamente nel segno. Fondandosi su esempi di giornali locali del 1989, Fabio Marri aveva osservato che badante "designa, nel linguaggio amministrativo e sindacale almeno della regione Emilia Romagna, la 'sorvegliante' addetta nelle strutture pubbliche all'assistenza di minori, anziani o inabili" (Fabio Marri, Giunte di lessico contemporaneo, in "Lingua nostra", LIII, I992, pp. I07-II9, a pp. Io8Iog). Il termine era stato ripreso nel GRADIT che lo qualificava come "burocratico" e nel LEI (V, c. 236) che lo riferiva appunto al romagnolo, anche se per origine e tipologia formativa non si tratta di un dialettalismo, ma di una voce dell'italiano di Romagna, voce presente ovviamente anche nel dialetto: abadant e abadanta compaiono infatti nel Vocabolario romagnolo-italiano di Antonio Mori (I840) con le accezioni di 'portinaio', 'servigiale', 'governante'. Tuttavia lo stesso Mori si fa scrupolo di precisare che "pero diconsi sempre relativamente a Convento di frati e di monache, a Spedali, Orfanotrofj e simili"; e per quanto riguarda la badante 'governante' si deve intendere la donna che "nelle Case de' Signori sta alla guardia de' bambini, e de' fanciulli": ospedali, conventi, case di signori, tutti luoghi più vicini alla lingua che al dialetto.

Non è facile stabilire con precisione il momento in cui la parola prese a circolare, dato che il modulo formativo che l'ha generata è piuttosto comune e sempre disponibile: fin dai primi secoli abbondano nomi di mestieri e 
professioni in -ante, siano participi presenti sostantivati (cantante, lavorante), siano formazioni che sfruttano quella terminazione come un suffisso produttivo con basi non solo verbali, ma anche sostantivali e aggettivali (cfr. Bruno Migliorini, I nomi del tipo "bracciante" [1936], in Id., Saggi linguistici, Firenze, I957, pp. I09-I28; La formazione delle parole in italiano, a cura di Maria Grossmann e Franz Rainer, Tubinga, 2004, pp. 212-214, 357-359). Migliorini aveva giustamente notato che la connessione del suffisso con l'-ante participiale "si riverbera anche sul significato dei nostri sostantivi come nomi di mestiere. In confronto con i nomi in -aio, (-aro), -iere, -(a)tore, -ino, ista, i nomi in -ante designano occupazioni meno stabili (-ante è più momentaneo) e perciò più modeste. Un bottegante è chi sta a bottega, vi serve e ne vive, mentre un bottegaio ha i suoi commessi, un caffeante è insieme "caffettiere e garzone di caffe" [...], un lavorante è meno stabile d'un lavoratore, un musicante è molto inferiore a un musicista" (p. I24). Nonostante ciò si possono invece trovare "gruppi di nomi in -ante perfettamente obiettivi" per singoli ambiti professionali o entro settori particolari, fra i quali Migliorini ricorda quello dei religiosi (frati barbanti, correggianti, scarpanti, zoccolanti, cercanti, mendicanti, questuanti): "la spinta sarà partita dal nome degli osservanti o da quello degli zelanti (i seguaci di S. Bernardino)". Un altro di questi settori è stato quello delle istituzioni assistenziali religiose, a cominciare da quelle rivolte al servizio degli infermi: nel gergo dei confratelli della fiorentina Misericordia vivono da secoli voci come giornante 'chi si occupa del trasporto degli infermi e dei defunti', questuante 'chi raccoglie elemosine', mutante 'chi assiste gli allettati e cambia loro vesti e fasce', nottante 'infermiere di notte'. Ma denominazioni simili, più o meno antiche e durevoli, si possono poi riscontrare in generale per il personale ospedaliero e infermieristico, e non solo in Toscana: barellante, faticante, praticante, punturante (cfr. Migliorini, p. I2I), tirocinante, turnante, volante 'infermiere che lavora senza turni fissi', ecc.

Anche badante, con molta probabilità, è un termine nato in questi ambiti: "Spedali, Orfanotrofj e simili" come dice il Mori, per la necessità onomasiologica di indicare una mansione particolare, seppur di carattere generico. E, lo si è visto, deve esser nato in Romagna, come confermano diverse attestazioni otto-novecentesche: "Ha l'obbligo [il curatore dell'ospizio dei trovatelli di Forli] di sorvegliare il mantenimento e l'educazione degli esposti in campagna [...]. Ad ogni piccolo rapporto spedisce la badante del pio luogo per esaminarli se sono lattanti" (Sesto Matteucci, Memorie storiche intorno ai Forlivesi benemeriti [...] e sullo stato attuale degli stabilimenti di beneficenza e d'istruzione in Forli, Faenza, I842, p. 22); "il piccolo Federico fu affidato alle mani di due donne francesi, Maria Duval e Madame de Rocoulle, le quali nel palazzo reale di Berlino disimpegnavano per tradizione quelle mansioni intermedie tra la bambinaia e la governante, che in Romagna sono affidate alla "badante"" (Alberto Savinio, Voltaire e Federico II [1945], in Id., Opere, a cura di L. Sciascia e F. De Maria, Milano, I989, p. 185).

Se questo è l'antefatto, bisogna tuttavia dire che fra l'antica "badante" delle Romagne e la "badante" dei nostri giorni ci corre come fra il giorno e la notte. E questo per la grossa rivoluzione che ha investito cultura, costumi, vita stessa della nostra società dopo la metà del secolo scorso. A cominciare dai suoi fondamenti ideali e materiali: il suo nucleo primigenio che era la famiglia, la religiosità che tutto pervadeva. Non più gli avvolgenti legami personali e di gruppo che facevan sì che nessuno restasse davvero isolato: nemmeno i trovatelli, non più grandi case accoglienti, ma alveari di piccoli appartamenti, non più domestici e cameriere e tutta una schiera di persone che provvedevano a ogni necessità, ma nuovi specialisti anonimi e lontani. Per venire al nostro caso, dopo la metà del secolo andarono via via scomparendo bambinaie, governanti, tate, servitori, segretari, dame di compagnia e tante altre figure un tempo familiari, lasciandosi dietro gusci di parole vuote, e non solo nelle "Case de' Signori". Ricordo che nel piccolo paese in cui crebbi e dove tutti vivevano in dignitosa povertà, ebbi la fortuna di sperimentare molte balie e di acquistare molti fratelli di latte, mentre i fratelli che vennero dopo, negli anni cinquanta e sessanta, furono allevati col latte in polvere, poiché di balie, a differenza delle lucciole che non son mai del tutto scomparse, non ce ne furono più. Anche la parola badante dovette attraversare quel radicale trapasso di civiltà, ma sulla sua sorte, negli ultimi decenni del secolo, gravarono due ulteriori fortissimi condizionamenti.

Da una parte il crollo demografico e il progressivo invecchiamento della popolazione, che orientò il settore d'azione della badante dal mondo infantile a quello dei vecchi e degli infermi. Già i due esempi giornalistici addotti da Marri ce ne mostrano un chiaro riflesso. Il primo, dalla "Cronaca di Bologna" della "Repubblica" del 20 gennaio 1989, fin dal titolo (Nelle elementari - A scuola una coop sostituirá le badanti) ci dice come stia definitivamente tramontando la figura della badante nella sua tradizionale accezione "romagnola". Il secondo, dalla "Gazzetta di Modena" del I8 novembre i989, intitolato Una "badante" per la notte, parla appunto del nuovo "fenomeno dell'assistenza privata all'interno degli ospedali modenesi", assistenza che un tempo era dovunque svolta da familiari o da infermiere professionali e suore appositamente incaricate. Nello stesso momento, 
proprio in relazione a questa nuova funzione di "pseudo-infermiera o assistente notturna", il termine badante comincia a diffondersi anche nel resto d'Italia: nel 1994, a proposito di una vicenda di «mercato nero dell'assistenza a pagamento, gestito da alcune "caporalesse"» scoperta in un ospedale di Vercelli, si parlò della piaga delle assistenze "mercenarie" abusive, svolte appunto dalle «cosiddette "badanti"» (Enrico De Maria, Infermiere" a luci rosse in ospedale, nella "Stampa" del I5 marzo 1994, p. I4). Nello stesso giornale, qualche mese più tardi, in una lettera del presidente del Collegio Provinciale Infermieri di Torino ("La Stampa", 26 settembre r994, p. 20), si denunciava il mercato sommerso di "fantomatiche associazioni che si pubblicizzano come infermieristiche" i cui soci, di fronte all'offerta di infermieri diplomati, temendo di esser soggetti a denunce per abuso della professione, avevano optato per la nuova denominazione: «Linventiva li ha ancora una volta soccorsi: ora si qualificano "badanti" e il quadro di riferimento concettuale è la "badanza"». Il neologismo, con tale nuova accezione, venne prontamente registrato nello Zingarelli del 1996: "Chi (o Che) assiste a pagamento degenti ricoverati in ospedali o case di cura".

Dall'altra il crollo del Muro di Berlino del 1989 che rese disponibile un'enorme massa di badanti a buon mercato. In pochi anni, dai paesi un tempo sotto l'influenza sovietica e in particolare dalla Romania e dalla Polonia, si riversarono in Italia fitte schiere di donne sicure di trovare un impiego e un alloggio, passando dal milione stimato nel 200 a al milione e seicentocinquantacinquemila del 20 22 (secondo un rapporto CENSIS del I4 maggio 20I2) e continuando ad aumentare, quasi senza flessioni, fino ai giorni nostri. Questo anche perché in Italia non solo sono aumentati gli anziani, ma si sono ulteriormente indebolite le reti familiari, il welfare è andato sfaldandosi, i ricoveri ospedalieri si sono accorciati, i servizi pubblici sono completamente assenti, insufficienti e costose le strutture per la lungodegenza.

Ecco così che la parola badante nel nuovo millennio ha assunto nuovo vigore e una nuova accezione, ritagliata questa volta sul tipo di donna dell'Europa dell'Est, priva di una preparazione specifica ma disposta ad ogni tipo di incombenza, assunta quasi sempre in modo irregolare attraverso il passaparola e spesso pagata male e a nero, quindi talora fonte di problemi e conflitti penosi: nei quali non si sa mai se a non amare e a non sopportare sia il badante o il badato, perché entrambi ignorano ormai il segreto rapporto che nel mondo di ieri legava fra loro padroni e servi, quando la religione illuminava ogni azione e insegnava ai padroni la cura e la responsabilità dei servi e ai servi la fiducia nei padroni. Tuttavia, al di là dei singoli e occasionali problemi, la "badante" del nuovo millennio svolge un lavoro prezioso e indispensabile, di cui dobbiamo tutti riconoscere il valore, perché senza di esso crollerebbe il difficile equilibrio su cui si regge l'attuale situazione sociale italiana.

Va anche aggiunto che solo con questa sua terza "mutazione genetica" la parola badante è entrata effettivamente nella lingua comune e nel fardello di parole che quotidianamente ci portiamo dietro. E questa volta si può anche individuare il momento preciso in cui è venuta alla ribalta: nei primi mesi del 2002, nelle discussioni giornalistiche relative alla nuova legge sull'immigrazione, la cosiddetta "Bossi-Fini", si fece infatti un gran parlare delle "badanti", le persone dell'Est che allora erano ancora "extracomunitarie", ma che, essendo impiegate nelle famiglie per sopperire a necessità impellenti e irrisolvibili in altro modo, andavano regolarizzate. Il termine fu subito preso per un neologismo giornalistico (del tutto ignota la preistoria "romagnola" e troppo recente e ancora non ben assestata la storia "italiana" della parola). Un neologismo spesso giudicato brutto o trattato con quel malcelato fastidio che accompagna di solito le novità lessicali. Bisogna però dire che in quel primo impatto su larga scala la parola serviva soprattutto di richiamo nei titoli dei giornali, mentre nell'uso comune era ancora scarsamente diffusa, quasi tutti preferendo continuare a impiegare, anche per le extracomunitarie accolte in casa per badare agli anziani, le solite denominazioni generiche (la donna, l'aiuto, e simili) o gli etnici di provenienza (la polacca, la rumena, ecc.). Tuttavia il fatto che la nuova particolare accezione del termine fosse recepita nel nuovo disegno di legge e che se ne parlasse largamente nei mezzi di comunicazione fini per sdoganare la parola, sulla quale venne subito appuntandosi la lente degli specialisti.

Gian Luigi Beccaria (Una parola neonata che piace poco. Non badate alla badante, nella "Stampa" del 27 aprile 20oz, p. 24), dopo aver ricostruito la storia della neoformazione e mostrato la sua fondamentale "normalità", così concludeva: «Ma perché questa parola piace così poco? Non è una di quelle "neutre" [...], tipicamente burocratiche, analgesiche, librate in un cielo immateriale. È così poco tecnocratica, sembra una parola alla buona. C'è chi vi sente un'aria spagnolesca, come l'antico "guardinfante". C'è chi invece trova "badante" quasi comico, ad alcuni sembra una parola poco seria, come di serie B, meno professionale. I motivi sono veramente tutti extralinguistici. Non piace per quel che ci sta dietro, la malattia, la vecchiaia. Non piace perché uno pensa 
che potrebbe averla inventata Bossi, o chi per lui, e allora le gira intorno un'aria deprofessionalizzante, sottilmente dequalificante: la "badante" non farà mai parte, come la "governante", veramente della casa. In realtà la parola, come tutte, in sé non è né bella né brutta».

Interessante fu anche l'intervento di Paolo Di Stefano (Lessico politico. "Badanti notturne": il sogno proibito che viene dall'Est, nel "Corriere della sera" del 2r aprile 2002, p. 33) che fra l'altro sosteneva che il termine era già apparso una prima volta sullo stesso quotidiano nel 1989 , indicando tuttavia in modo chiaro la nuova accezione: «sdoganate ormai dai documenti ufficiali alla cronaca di giornale, le "badanti" sono (e saranno) soltanto le "assistenti familiari" immigrate (per lo più provenienti dai Paesi dell'Est europeo)». Per eliminare la "sgradevole assonanza" con parole come badilante o benandante, Di Stefano suggeriva inoltre alcuni sostituti: assistente familiare, ausiliaria, colf, vegliante. Si tratta di termini che combaciano solo in parte con la semantica di badante e che hanno tutt'altra storia, ma, al di là di ciò, cercare di sostituire a freddo delle parole sgradite è un'operazione quasi sempre vana: il neologismo non fu allora minimamente scalzato, non lo è adesso che neologismo non è più e, continuando questo stato di cose ovvero l'invecchiamento della popolazione, lo sarà difficilmente anche in futuro.

Oggi badante è una voce del tutto acclimatata, non solo nella lingua comune, ma in letteratura e nel cinema (compare sovente fin nei titoli). E il fenomeno dell'impiego delle badanti è ampiamente trattato dalla sociologia $\mathrm{e}$ in tanti altri settori del discorso pubblico. Certo la parola mantiene una connotazione non pienamente positiva per diverse ragioni, ma innanzitutto perché continua a indicare in modo generico chi, senza una specifica preparazione, presta assistenza anche in casi in cui magari ci vorrebbe. Proprio perciò più di una volta si è pensato di riqualificare tale lavoro attraverso una formazione professionale del badante, in modo da trasformarlo in un assistente domiciliare dotato di competenze infermieristiche e di una sua professionalità. Ed è comprensibile che la riqualificazione punti anche sulla scelta di una nuova denominazione, per distinguere la vecchia generica mansione del badare un anziano, dalla nuova professione infermieristica. Così si spiegano le affermazioni perentorie della lettera citata da Marazzini; e anche ciò che di tanto in tanto si legge sui giornali, in parte esemplificato anche da Giovanni Adamo e Valeria Della Valle alla voce assistente familiare nei loro Neologismi. Parole nuove dai giornali (Roma, 20o8). Fra codesti esempi merita citare l'ultimo, dal "Corriere della sera" del I7 febbraio 2007: «Addio al termine badante [...] Non più "badante", ma "assistente familiare" o "addetta alla cura della persona": nel nuovo contratto delle colf sparisce il termine ancora usato nel linguaggio comune e sui permessi di soggiorno». Ma, come si è accennato, per il vero "addio", se mai ci sarà, ci vorrà ancora del tempo.

\section{Cita come:}

Massimo Fanfani, Ancora a proposito dibadante , "Italiano digitale", 2019, VIII, 2019/1 (gennaiomarzo), pp. 77-80.

DOI: $10.35948 / 2532-9006 / 2019.3129$

\section{Copyright 2019 Accademia della Crusca} Pubblicato con licenza creative commons CC BY-NC-ND 